\title{
N-acetylcysteine in Severe COVID-19: The Possible Mechanism
}

\author{
Md Jahidul Hasan (iD) ${ }^{1}{ }^{*}$ \\ ${ }^{1}$ Clinical Pharmacy Services, Department of Pharmacy, Square Hospitals Ltd., 18/F Bir Uttam Qazi Nuruzzaman Sarak, West Panthapath, Dhaka-1205, Bangladesh \\ "Corresponding author: Clinical Pharmacist (Critical Care), Clinical Pharmacy Services, Department of Pharmacy, Square Hospitals Ltd., 18/F Bir Uttam Qazi Nuruzzaman Sarak, \\ West Panthapath, Dhaka-1205, Bangladesh. Email: jahidul@squarehospital.com
}

Received 2020 June 12; Revised 2020 August 05; Accepted 2020 August 23.

Keywords: Inflammation, N-acetylcysteine, COVID-19

\section{Dear Editor,}

Coronavirus Disease 2019 (COVID-19) caused by Severe Acute Respiratory Syndrome Coronavirus-2 (SARS-CoV-2), a new beta coronavirus, was officially declared a global pandemic by the World Health Organization (WHO) on March 11,2020 , after coming under the spotlight through a pneumonia outbreak in Wuhan, China, first reported on December 30, 2019. As of June 2, 2020, 6,194,533 confirmed COVID-19 cases, and 376,320 associated deaths were reported worldwide (1). The clinical investigation of COVID19 cases shows that patients with COVID-19 are admitted to hospitals with common viral pneumonia symptoms, mostly including fever, dry cough, fatigue, sore throat, and shortness of breath (2). Hyperinflammation-associated Acute Lung Injury(ALI) is the leading cause of death among patients with severe COVID-19, and highly expressed inflammatory cytokines, including interleukin (IL)-6, IL-10, IL-1 $\beta$, and Tumour Necrosis Factor- $\alpha$ (TNF- $\alpha$ ) play a pivotal role in aggravating the inflammatory responses $(3,4)$.

SARS-CoV-2 binds to the Angiotensin-Converting Enzyme-2 (ACE-2) receptor in human alveolar epithelial cells to enter the host cell; in response, the activated innate and adaptive immune systems of the host release a variety of pro-inflammatory cytokines, including IL-6, IL-10, TNF- $\alpha$, and MIP-1- $\alpha$, leading to a cytokine storm. In addition, the released chemokines activate the secretion of other inflammatory mediators from peripheral blood into the site of inflammation and potentiate the cytokine storm (Figure 1). Increased vascular permeability and fluid accumulation in alveoli is due to the activities of these proinflammatory cytokines, resulting in respiratory failure $(3,5)$.

The WHO declares N-acetylcysteine (NAC), a relevant medication intended to use in the basic health system, and it is generally used as a mucolytic, anti-inflammatory, and antioxidant agent (6). As an anti-inflammatory agent,
NAC prevents the release of pro-inflammatory cytokines in the initial state of immune proliferation in disease progression, and this mechanism is based on the inhibition of the activities of lipopolysaccharides. Nuclear Factorkappa B (NF- $\kappa \mathrm{B})$ plays a pivotal role in inflammation cascades, and the bonding between I- $\kappa \mathrm{B}$ kinase (I- $\kappa \mathrm{B})$ and NF$\kappa \mathrm{B}$ prevents its nuclear translocation. $\mathrm{I} \kappa \mathrm{B}$ kinase $\beta$ (IKK $\beta$ ) regulates the separation of $\mathrm{I}-\kappa \mathrm{B}$ from NF- $\kappa \mathrm{B}$ by phosphorylation (lipopolysaccharide-induced) and its degradation by the proteasome and allows NF- $\kappa \mathrm{B}$ to enter into the nucleus where this transcription factor (NF- $\kappa \mathrm{B}$ ) activates the release of pro-inflammatory cytokines, mostly including IL-6, IL-1 $\beta$, and TNF- $\alpha$ (6), which are also found in high levels in patients with severe COVID-19 $(3,4)$. NAC exhibits its sole anti-inflammatory characteristic by inhibiting the activation of NF- $\kappa$ B activities through the suppression of the activities of lipopolysaccharides (Figure 1). Multiple studies found high serum IL-6 levels in COVID-19 patients (3-5), and its release is significantly enhanced by the action of both IL-1 $\beta$ and TNF- $\alpha$ in early inflammation state. In effect, NAC inhibits the expression of IL- 6 by significantly reducing the levels of IL-1 $\beta$ and TNF- $\alpha(3,7,8)$. Furthermore, in severe COVID-19 patients, high levels of IL-10 are detected $(3,5)$, which significantly contributes to immune proliferation. An in-vitro study showed that IL-10 mRNA expression was highly downregulated by NAC, resulting in the suppression of the proliferation of inflammation cascades (7, 8).

In its primary role, NAC is a precursor of glutathione (GSH) synthesis, which is the principal antioxidant that the body produces to protect its cells from serious injury or death due to high levels of Reactive Oxygen Species (ROS), resulting in intracellular oxidative stress (6). In addition, inflammation extensively accelerates the production of ROS, leading to massive oxidative stress at the site of inflammation (Figure 1). An increased level of TNF- $\alpha$ al- 


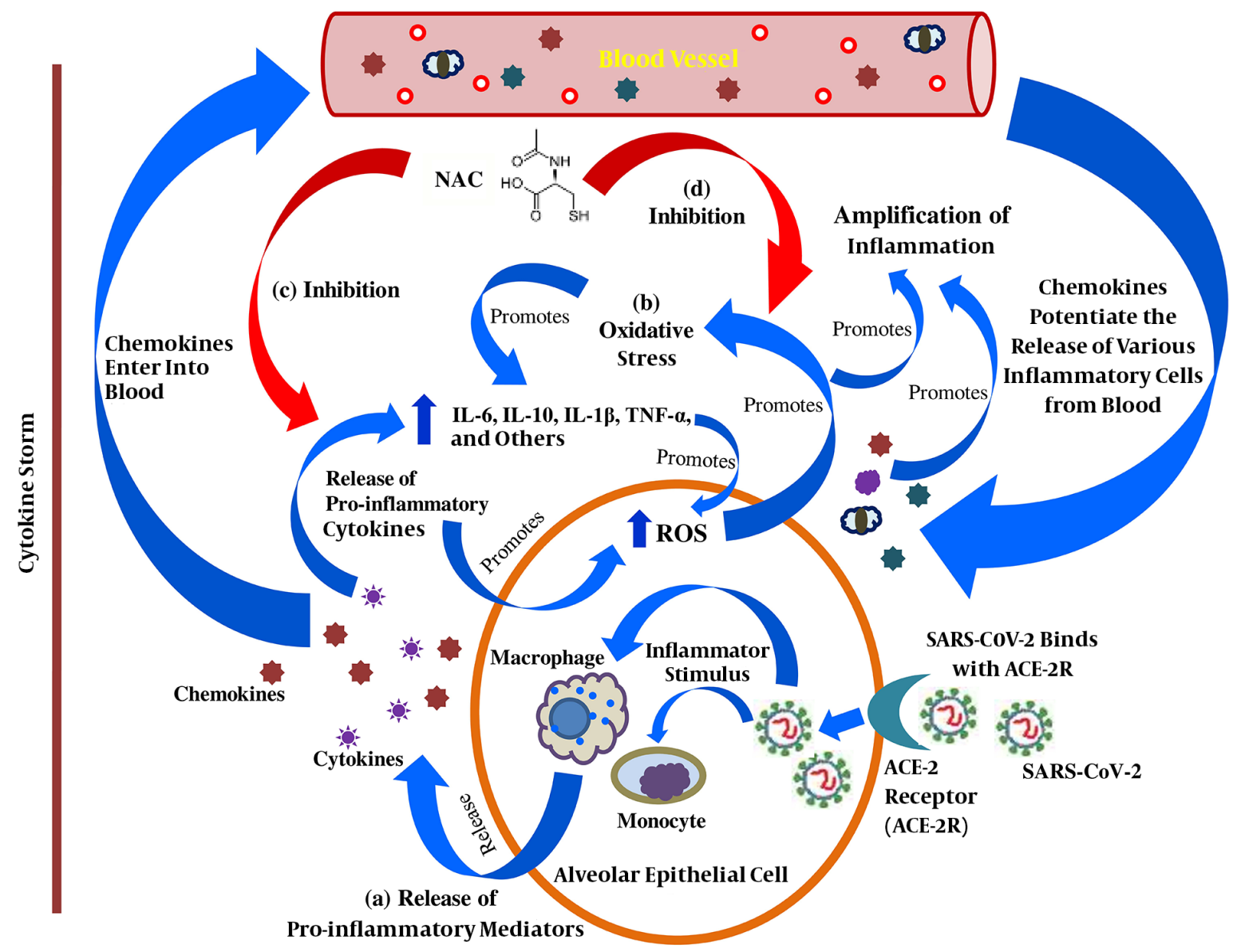

$\mathrm{IL}$, Interleukin; TNF- $\alpha$, tumour necrosis factor- $\alpha$; NAC: N-acetylcysteine; MIP-1 $\alpha$, macrophage inflammatory protein-1 $\alpha$; ROS, reactive oxygen species

Figure 1. Anti-inflammatory role N-acetylcysteine in patients with severe COVID-19; (a) Release of pro-inflammatory cytokines in direct response to SARS-CoV-2-host cell interaction; (b) Proliferation of intracellular oxidative stress due to the release of pro-inflammatory cytokines; (c) Inhibitory role of NAC in the extensive release of pro-inflammatory cytokines and chemokines; (d) Inhibitory role of NAC in the exacerbation of intracellular oxidative stress

lows more ROS to generate in mitochondria, which significantly potentiates NF- $\kappa$ B to exacerbate the cytokine storm, leading to cell death (9) (Figure 1). In addition, in response to oxidative stress, lung epithelial cells predominantly release pro-inflammatory cytokines, including IL6 , IL-10, IL-1 $\beta$, and TNF- $\alpha$ (Figure 1). NAC improves the cellular total antioxidant capacity through improving GSH concentration and directly interacts with ROS, including hydroxyl radicals, nitrogen dioxide, and carbon trioxide ion using its free thiol side-chain (8). Even at a low intracellular concentration, NAC replenishes the GSH level, resulting in a healthy intracellular environment $(8,9)$. The lower the level of intracellular oxidative stress, the higher the chance of overcoming pulmonary damage in severe COVID-19 infection(9). Thus, NAC inhibits the expression of pro-inflammatory cytokines, which may potentially suppress the progression of ALI in patients with severe COVID19.

An extensive release of pro-inflammatory cytokines and excessive intracellular oxidative stress in a severe stage of COVID-19 causes massive cytokine storm that develops severe ALI, leading to respiratory failure (2-5). The multi-dimensional target-specific anti-inflammatory roles of NAC significantly suppress the release of IL-6, IL-10, IL-1 $\beta$, and TNF- $\alpha$, which are the most robust components of inflammation cascades, and the primary antioxidant prop- 
erty of NAC reduces the intensity of intracellular oxidative stress in severe COVID-19 (7-9). Therefore, NAC may be effective in the management of severe pulmonary inflammation in viral pneumonia, including COVID-19, and may reduce the risk of acute respiratory failure-associated mortality in patients with severe COVID-19.

\section{Footnotes}

Conflict of Interests: The author declares no conflict of interest.

Funding/Support: None.

\section{References}

1. World Health Organization. Coronavirus disease 2019 (COVID-19) situation report-134. 2020, [cited 2020 June 3]. Available from: https://www.who.int/docs/default-source/coronaviruse/situationreports/20200602-covid-19-sitrep-134.pdf.

2. Verity R, Okell LC, Dorigatti I, Winskill P, Whittaker C, Imai N, et al. Estimates of the severity of coronavirus disease 2019: a modelbased analysis. The Lancet Infectious Diseases. 2020;20(6):669-77. doi: 10.1016/s1473-3099(20)30243-7.

3. Zhang $\mathrm{C}, \mathrm{Wu} \mathrm{Z}$, Li JW, Zhao H, Wang GQ. Cytokine release syndrome in severe COVID-19: interleukin-6 receptor antagonist tocilizumab may be the key to reduce mortality. Int $J$ Antimicrob Agents. 2020;55(5):105954. doi: 10.1016/j.ijantimicag.2020.105954. [PubMed: 32234467]. [PubMed Central: PMC7118634].

4. Huang C, Wang Y, Li X, Ren L, Zhao J, Hu Y, et al. Clinical features of patients infected with 2019 novel coronavirus in Wuhan, China. The Lancet. 2020;395(10223):497-506. doi: 10.1016/s0140-6736(20)30183-5.

5. Fu Y, Cheng Y, Wu Y. Understanding SARS-CoV-2-Mediated Inflammatory Responses: From Mechanisms to Potential Therapeutic Tools. Virol Sin. 2020;35(3):266-71. doi: 10.1007/s12250-020-00207-4. [PubMed: 32125642]. [PubMed Central: PMC7090474].

6. Tardiolo G, Bramanti P, Mazzon E. Overview on the Effects of NAcetylcysteine in Neurodegenerative Diseases. Molecules. 2018;23(12). doi: 10.3390/molecules23123305. [PubMed: 30551603]. [PubMed Central: PMC6320789].

7. Palacio JR, Markert UR, Martinez P. Anti-inflammatory properties of N-acetylcysteine on lipopolysaccharide-activated macrophages. Inflamm Res. 2011;60(7):695-704. doi: 10.1007/s00011-011-0323-8. [PubMed: 21424515].

8. Rocksen D, Lilliehook B, Larsson R, Johansson T, Bucht A. Differential anti-inflammatory and anti-oxidative effects of dexamethasone and $\mathrm{N}$-acetylcysteine in endotoxin-induced lung inflammation. Clin Exp Immunol. 2000;122(2):249-56. doi: 10.1046/j.1365-2249.2000.01373.x. [PubMed: 11091282]. [PubMed Central: PMC1905762].

9. Zhang Q, Ju Y, Ma Y, Wang T. N-acetylcysteine improves oxidative stress and inflammatory response in patients with community acquired pneumonia: A randomized controlled trial. Medicine (Baltimore). 2018;97(45). e13087. doi: 10.1097/MD.0000000000013087. [PubMed: 30407312]. [PubMed Central: PMC6250560]. 